\title{
REVISIONES
}

\section{Tendencias curriculares en educación química (1950-1990)}

\author{
Curriculum trends in chemistry education (1950-1990)
}

Tendências curriculares em educação química (1950-1990)

\begin{abstract}
Nora Faúndez-Vallejos
Grupo de estudos e pesquisas sobre formação de educadores (GEPEFE), Faculdade de Educação, Universidade de São Paulo, Brasil, 55(19)3231 1201, nojfava@hotmail.com
\end{abstract}

\begin{abstract}
Este artículo se sitúa en el área de la historia de la educación científica, y en él son discutidas las principales tendencias curriculares en educación química, desde la década de 1950 hasta 1990, considerando cuatro elementos: los objetivos, la selección y organización de los contenidos, los principios de organización del proceso de enseñanza-aprendizaje de la química y los procedimientos de evaluación.

Corresponde a una revisión de la literatura destacada de ese periodo, que da a conocer el desarrollo y la evolución que estos elementos han presentado en la educación científica y específicamente en la educación química. Se demuestra la evolución que ha tenido la educación científica, en esas décadas, al enfatizar en la construcción social del conocimiento científico y una educación como formación integral.
\end{abstract}

RESUMEN

Palabras clave: educación científica, educación química, formación integral

\section{ABSTRACT}

This article is related to the history of science education, discussing the main curricular trends in chemistry education from 1950 to 1990, and considering four elements: objectives; selection and organization of content; principles of organization of chemistry teaching-learning; and grading procedures. The study is based on a review of the relevant literature for that period on the development and evolution of these elements in science education, and specifically in chemistry education. The developments that took place in science education in that period are discussed, emphasizing the social construction of scientific knowledge and education.

Keywords: Science education, chemistry education, comprehensive training

\section{RESUMO}

Situa-se na área da história da educação científica e são discutidas as principais tendências curriculares em educação química de 1950 a 1990, considerando quatro elementos: os objetivos, a seleção e a organização dos conteúdos, os princípios de organização do processo de ensino-aprendizagem da química e os procedimentos de avaliação. É realizada uma revisão da literatura desse período, o que permite conhecer o desenvolvimento e a evolução que estes elementos têm apresentado na educação científica e, particularmente, na educação química. Evidencia-se a evolução da educação científica nesse período ao enfatizar a construção social do conhecimento científico e uma educação como formação integral.

Palavras-chave: educação científica, educação química, formação integral 


\section{INTRODUCCION}

Interesada en la historia de la educación científica, presento parte de un referencial teórico (de una investigación más amplia) que discute las principales tendencias curriculares en la enseñanza de la química entre los años 1950 y 1990 (Faúndez-Vallejos, 1993). Este referencial fue elaborado a partir de algunas consideraciones de la literatura de este período, utilizando referencias de esas décadas, para investigar cuatro componentes curriculares, discutiendo el desarrollo y la evolución que estos han presentado en un período determinado.

En este sentido, en lo que se refiere a algunos componentes del currículum, presento a continuación sus principales tendencias en el ámbito de la educación en ciencias, con particular énfasis en la educación química, considerando: los objetivos de la enseñanza, la selección y organización de los contenidos, los principios del proceso de enseñanzaaprendizaje y los procedimientos de evaluación. De esta manera, pretendo contribuir, compartiendo, con los profesores de química (y con aquellos que se encuentran en proceso de formación inicial), una visión general de los esfuerzos realizados por los investigadores del área durante esas décadas, con el fin de mejorar la enseñanza en esta área.

\section{OBJETIVOS DE LA ENSEÑANZA DE LA QUÍMICA}

Los objetivos, junto con la evaluación, constituyen el elemento curricular más importante del cual dependerán los otros. Corresponden al punto de partida del proceso de desarrollo curricular, al determinar las posibles opciones que serán tomadas en consideración con relación a otros elementos curriculares.

En la década de 1950, los estudios en educación científica critican los objetivos curriculares en ciencias, considerándolos inapropiados al no proponer una ciencia para todos y ser predefinidos, dando como resultado cursos académicos y abstractos (Hodson y Reid, 1988).

A partir de 1960, son elaborados proyectos curriculares que son considerados 'Cursos modernos de ciencias' en oposición a los cursos tradicionales existentes, que se caracterizaban por ser muy extensos, descriptivos, enfatizando la acumulación de informaciones y el uso de demonstraciones experimentales que tenían como objetivo confirmar lo enseñado en la teoría (Pode, 1967). Contrario a estas características, los proyectos modernos de ciencias -entre los que se sitúan, para la enseñanza de la química, el CHEMS-Química: una ciencia experimental; el CBA-Sistemas Químicos y el Nuffield- intentan enfatizar, según Klopfer (1971): la naturaleza y la estructura de la Ciencia (Química) y los procesos de investigación científica; la diferencia entre investigación e interpretación, entre resultados y esquemas conceptuales; la profundidad y no la extensión del contenido; el uso de laboratorios para introducir, explorar y sugerir problemas; el uso de las investigaciones como base para el desarrollo del curso y, las discusiones en la sala de aula.

Estos énfasis denotan la preocupación por alcanzar un equilibrio contenido-proceso, lo que caracteriza los programas curriculares de Química hasta el final de la década de 1970 (Frazer, 1979).

Para dar continuidad a esta tendencia, a través del programa para el mejoramiento de la enseñanza, la UNESCO propone los siguientes objetivos como siendo esenciales al 
pensar en cualquier propuesta de educación en ciencias: la realización de experimentos por parte de los alumnos que los capacite para la adquisición de conocimientos; el desarrollo de habilidades y de actitudes relevantes para el estudio y la práctica de la ciencia, y su aplicación en lo cotidiano; el estímulo por la curiosidad, y el interés por la ciencia y sus métodos de investigación; y el interés y los cuidados del medio ambiente (Kempa, 1986).

Según Santos (1991), el equilibrio contenido-proceso, tal como era abordado o pretendido en esos proyectos, puede ser criticado por el hecho de mitificar el método científico, pretender hacer de los alumnos pequeños científicos, y de enfatizar el inductivismo y el aprendizaje por descubrimiento. Estas críticas, junto a los resultados poco promisores de la evaluación de estos proyectos en términos de aprendizaje de los alumnos, llevan a la comunidad de educadores en ciencias a repensar la concepción del proceso de enseñanza-aprendizaje de ciencias.

Para este autor, el alumno ya no es más considerado como un sujeto que recibe el saber construido, sino que lo construye activamente de acuerdo con la realidad, a partir de sus ideas previas, creencias y valores. En este sentido, el aprendizaje de las ciencias implica un cambio conceptual y, consecuentemente, la enseñanza de las ciencias y de la química, son considerados como procesos que deben estimular ese cambio. Surge así, en las propuestas curriculares para la enseñanza de las ciencias, elaboradas a partir de la década de 1980, la adopción de enfoques constructivistas asociados a la formación de la ciudadanía. En este contexto, en el que se enfatizan las necesidades de la sociedad y se concibe al alumno como sujeto constructor de conocimientos, Kronhauser (1980) menciona que la Química debe ser concebida y usada para ayudar a las personas a entender la naturaleza y el medio, para así usar sus posibilidades, resolver sus propios problemas y tomar decisiones. Estos aspectos son considerados en propuestas que surgen del movimiento CTS (Ciencia, Tecnología y Sociedad) para la enseñanza científica, que persigue los siguientes objetivos:

- comprender la naturaleza de la ciencia y su papel en la sociedad, lo que implica tener conocimientos básicos sobre los principios de la Filosofía y la Historia de la ciencia con relación a las limitaciones del conocimiento científico;

- construir conocimientos y elaborar interpretaciones acerca de los productos químicos y de cómo estos interactúan en nuestras vidas.

Las propuestas, para las décadas siguientes en la enseñanza de la Química tienen por objetivo: la construcción del conocimiento químico por parte de los alumnos a partir de sus ideas previas, promoviendo el cambio conceptual para contribuir con una formación como ciudadanos; habilitándolos para tomar decisiones de naturaleza científica, tecnológica y social y, así, prepararlos para la vida.

Según Kelly (1981), se trata de adoptar un enfoque menos rígido de la especificación de los objetivos (respecto a cómo era considerado en la década de 1950 y 1960), considerándolos provisorios y abiertos a constantes modificaciones y reformulaciones.

\section{SELECCIÓN Y ORGANIZACIÓN DE LOS CONTENIDOS}

Los contenidos no constituyen fines en sí mismo, pero sí, medios que permiten desarrollar capacidades, más que acumular informaciones, para la obtención de los objetivos propuestos. 
En la década de 1950, la ciencia era fundamentalmente concebida como un cuerpo de hechos y principios, considerando como correctas todas las ideas aceptadas por la comunidad científica (Ashman, 1985). Esto era transmitido a los alumnos bajo un enfoque tradicional, con conocimientos lógicamente estructurados, como algo estático y acabado, sin considerar criterios tales como aspectos lógicos, históricos y psicológicos. Según Moreira y Axt (1987), tal enfoque enfatizaba la importancia de la estructura de la ciencia, o sea, el funcionamiento intelectual en su desarrollo, considerando que 'Ciencia es eso', un modelo curricular como concepción científica, o el currículum como racionalismo académico.

Respecto al trabajo de laboratorio, actividad importante en la educación científica, este se basaba en un enfoque ilustrativo, donde los experimentos tenían la función de comprobar la teoría. Es decir, eran realizadas demostraciones de aquello que los alumnos ya sabían, enfatizando la memorización, reforzando la teoría con evidencias experimentales y presentándolas como un producto (Blizzard et al., 1975).

Frente a esto, las universidades critican severamente los cursos de Química en la enseñanza secundaria por ser poco actualizados, tradicionales, extensos y priorizando el uso de libros de texto; que de acuerdo a Pode (1967), era considerado como una enciclopedia llena de información esencial. Parece que la atención estaba en cumplir un programa, transmitiendo un contenido como si nada más hubiera, sólo el conocimiento científico, aislado de la realidad, con un enfoque tradicional de la Ciencia; lo que resultaba impersonal y sin implicar responsabilidad social, llevando a los alumnos a tomar distancia de esta área de estudios (Hodson y Reid, 1988). Todo esto llevó a una completa revisión de los programas y, así, la década de 1960-1970 se caracterizó por un cambio de paradigma, de una enseñanza de ciencias entendido como un cuerpo establecido de conocimientos para una enseñanza de ciencias, concebido como un método para generar y validar esos conocimientos (Caamaño, 1988). Surge un movimiento general de ciencias, con el interés de educar a través de la ciencia y no en ciencias, lo que da una nueva visión de la ciencia en las escuelas; cambiando el currículum científico, lo que influyó enormemente en la enseñanza de la química.

Debido a la proliferación de conocimientos químicos, así como a la necesidad de que la escuela fuera relevante frente a las necesidades de los alumnos, se priorizó la reducción de los contenidos de química escolar así como un mayor énfasis en los procesos de la química (Ashman, 1985), diferente a lo que era considerado en ese momento: cubrir un gran número de tópicos. En el nuevo currículum se enfatizaba la profundización de las relaciones entre diferentes contenidos. Y, ¿cómo seleccionar esos contenidos? Kelly (1981) menciona tres aspectos:

- Teoría del conocimiento: teorías epistemológicas de la naturaleza del conocimiento e ideas sobre la naturaleza de las ciencias;

- $\quad$ Sociedad: visión de la sociedad y de la cultura;

- Necesidades: modos a través de los cuales estas consideraciones forman parte de la preocupación de la planificación del currículum.

Se trataba de saber seleccionar los contenidos adecuados que proporcionaran una visión actual de la ciencia, fueran accesibles a los alumnos y susceptibles de interesarles (Gil Pérez et al., 1991). Esta concepción cognitiva del currículum apunta a la importancia de tomar en 
cuenta el desarrollo de los procesos cognitivos, habilidades básicas y fundamentales para toda actividad científica (Moreira y Axt, 1987). Algunas de sus características eran:

- centrarse en el alumno y en el proceso de aprendizaje en sí (el "cómo" de la educación), y no simplemente en el contexto social más amplio;

- desarrollar en el alumno cierta autonomía intelectual, lo que le permitiría realizar sus propias interpretaciones en las situaciones fuera del contexto educativo.

El laboratorio, por otro lado, era considerado como una característica clave del currículum (Waddington, 1980), ya que permitiría un balance adecuado entre teoría y práctica. Por lo tanto, las experiencias de laboratorio y la enseñanza expositiva no debían ser tratadas independientemente, sino de forma coordinada.

Este tipo de trabajo de descubrimiento inductivo o hipotético-deductivo, enfatizaba más la estructura del conocimiento que la estructura mental del alumno que aprende, considerando el método científico como siendo el método correcto o adecuado. Esta perspectiva empírico-inductiva de la ciencia es característica de los proyectos de los años de 1960-1970, en los cuales el conocimiento tiene como fundamentación la experiencia. Lo que se proponía era un enfoque investigativo, explorando, sugiriendo e introduciendo problemas en lugar de confirmar y tratar apenas los conocimientos específicos. Se trataba de enfatizar el desarrollo de diferentes habilidades, tanto para la ciencia como para el día a día, de manera tal que los alumnos aprendieran acerca de la importancia de los contenidos para sus vidas. En ese sentido, la participación directa de los alumnos en la experimentación permitiría el desarrollo de conceptos y alcanzar un aprendizaje significativo (Waddington, 1980; Buchweitz, 1985).

A comienzos de los años 1980, comienza a surgir el interés por el contexto en el que se ubica la ciencia y se comienza a relacionar la educación en ciencias con aspectos prácticos, culturales y sociales (Caamaño, 1988). Por ejemplo, el impacto de la química en el medio ambiente, analizando la contaminación y la industria petroquímica.

En esa década, se publica el documento 'Science Educaction in School', que se refería al currículum científico para las décadas siguientes, indicando que debía estar dirigido a todos los alumnos de la enseñanza secundaria (Science for all) y con un enfoque de ciencia integrada. Un enfoque práctico, considerando situaciones de soluciones de problemas y necesidades para enfrentar asuntos tecnológicos de la vida real.

También en esos años, la American Chemical Society, con apoyo financiero de la National Science Foundation, planificó desarrollar un proyecto interdisciplinario de Química denominado "Química para la Comunidad" (CHEMCOM), el cual debería ser adoptado en las escuelas a partir de 1990 (Ware et al., 1986). Es más, el CHEMCOM, fue desarrollado para formar ciudadanos dentro de una concepción de ciencia, tecnología y sociedad (CTS), considerando el papel de la química en sus vidas personal y profesional.

La organización y la implantación de este movimiento mundial de CTS surge en la década de 1970, a raíz del impacto de la ciencia y de la tecnología en la sociedad moderna, por lo tanto, en la vida de las personas, lo que llevó a la necesidad de que los individuos adquirieran conocimientos científicos. Este movimiento tiene por objetivo entregar a los alumnos conocimientos que les permitan participar de esta sociedad, en el sentido de buscar alternativas de aplicaciones de la ciencia y de la tecnología dentro de la visión de bienestar social. De acuerdo a Hostetter (1985), los contenidos de los cursos para formar ciudadanos 
debían considerar métodos y conocimientos científicos, principios químicos, química descriptiva direccionada para el ciudadano, problemas sociales y toma de decisiones. Para eso, los contenidos debían estar determinados por los siguientes criterios:

- $\quad$ ser relevantes para el medio local;

- estimular el interés de los alumnos;

- $\quad$ estar en el ámbito de las competencias de los profesores.

Así, era importante incluir temas de CTS en el currículo, ya que a través de éstos se podría promover el desarrollo de actitudes de toma de decisiones, producto de discusiones y de la comprensión de aspectos sociales. Desde esa perspectiva, los temas CTS considerados como relevantes eran: Salud, alimentos y agricultura, recursos energéticos, tierra, agua y recueros minerales, industria y tecnología, medio ambiente, transferencia de información y tecnología y, ética y responsabilidad social.

Dentro de este contexto, el CHEMCOM se basa en la suposición de que el interés del alumno por ciertos aspectos sociales que se relacionan con la química es anterior al interés por la propia química. Por lo tanto, la organización del currículum depende de la definición de Química que los alumnos necesitan conocer para comprender asuntos sociales específicos. Es decir, la química que se enseñaría estaría determinada por la selección de los temas (Ware et al., 1986). Los principales contenidos que CHEMCOM proponía eran: importancia del agua, la conservación de los recursos químicos, petróleo, la química nuclear en nuestro mundo, química, aire y clima, química y salud e, industria química.

Hacia la década de 1980, en general, se consideraba importante desarrollar el pensamiento crítico y un mejor entendimiento y apreciación de la ciencia, a través del currículum. Para eso, según Caamaño (1988), el conocimiento debería desarrollarse considerando la ciencia como una actividad cultural para el ciudadano. Por lo tanto, resulta necesario contextualizar la enseñanza de las ciencias (química), de tal manera que los alumnos puedan desarrollar una compresión más amplia de la ciencia química y de los procesos científicos. En otras palabras, la acción de la enseñanza no podía quedar relegada únicamente a los aspectos relacionados con la lógica interna de la disciplina, valorando y caracterizando el conocimiento de datos y de teorías, sin considerar aspectos psicológicos, históricos y sociológicos en lo que se refiere a la construcción del conocimiento científico y a su interacción con el medio ambiente.

En este contexto, frente a la necesidad de que la enseñanza de las ciencias esté más contextualizada y que los profesores estén mejor preparados, Matthews (1990) propone incluir Historia y Filosofía de la Ciencia en los cursos de formación de profesores de ciencias (Química), ya que al considerar aspectos históricos, filosóficos y sociológicos en las aulas, se contribuiría a humanizar los contenidos, mejorar la comprensión de los conceptos científicos, comprender el método científico (es decir, la naturaleza de la ciencia), tornar la idealización de la ciencia más humana y comprensible, y la enseñanza coherente, critica y motivadora. Esto ayudaría al alumno a entrar en el mundo de la ciencia. Para ello se sugería una selección de contenidos que fuesen relevantes, abiertos y con significado teórico y social, esto, sin descuidar los intereses de los alumnos como punto de partida.

En términos de contenidos químicos, se sabe que la cantidad de información aumenta contínuamente, por lo tanto no hay posibilidad de enseñar más que una pequeña fracción de ese conocimiento, la que debe ser tratada de la manera más eficiente posible, seleccionando 
contenidos que den una visión positiva de la ciencia y a los cuales los alumnos puedan tener acceso. Se trata de contenidos que permiten pensar en problemas químicos que los alumnos podrán encontrar más tarde en sus profesiones y que formen parte de asuntos o aspectos sociales importantes. En términos químicos específicos, se debían desarrollar cursos con una visión realista y balanceada de la química moderna para propiciar en los alumnos un conocimiento útil de la química factual y una actitud crítica respecto a las teorías (Gillespie y Humphreys, 1972). Corresponde al profesor estimular al alumno para que comprenda que las teorías generalmente son creadas para la explicación de hechos, ya que cuando el alumno no tiene la posibilidad de comprobar la aplicación de éstas, no aprende lo que la química realmente es y el papel que tiene en el mundo moderno. Interesa la calidad y la forma en que son tratados estos contenidos y que permitan el desarrollo de habilidades.

Finalmente, para las décadas siguientes se propone considerar la Química en la sociedad moderna, la influencia en la industria local y la interacción y cooperación entre industria, escuela y universidad (Gillespie y Humphreys, 1972; Hodson y Reid, 1988; Ware et al., 1986).

\section{PRINCIPIOS DE ORGANIZACIÓN DEL PROCESO DE ENSEÑANZA- APRENDIZAJE DE LA QUÍMICA}

En las primeras décadas del período en estudio, el método de enseñanza era rígido, enfatizando una instrucción formal, lo que llevaba a tener alumnos pasivos con una gran acumulación de conocimientos. Esto implicaba un énfasis en la memorización de informaciones. A partir de los años 1980, surge la necesidad de adoptar métodos activos de aprendizaje, con el objetivo de ayudar al alumno en la construcción de ideas. En esa época, surge la renovación en la concepción epistemológica de lo que es la ciencia (con Popper, Kuhn, Lakatos y Feyerabend) y un avance en la psicología cognitiva, que entiende el aprendizaje como una construcción (Caamaño, 1988). Según Hernández (1990), diferentes teorías psicológicas del proceso de enseñanza-aprendizaje comparten principios constructivistas, señalando que el desarrollo y el aprendizaje son básicamente resultados de un proceso de construcción.

Frente a esta situación, a partir de esos años se adopta, como marco referencial psicopedagógico, una concepción constructivista del proceso de enseñanza-aprendizaje que asume al alumno como constructor de ideas (Hodson y Reid, 1988). Como ya fue mencionado, surge la necesidad de utilizar métodos activos de aprendizaje que darán al alumno oportunidades para explorar y reconstruir ideas. Una enseñanza adecuada implicaba que el alumno llegara al nuevo contenido considerando alguna estructura existente de ideas sobre éste, y a partir de eso, ayudarlo en el desarrollo y el cambio de estructura de conocimiento ya existente, para así ir construyendo la ciencia (Frazer, 1984). Según esta perspectiva, el profesor era un mediador entre el conocimiento científico y la construcción de los alumnos, y por eso, debía poseer un dominio conceptual que le permitiese sugerir las actividades más apropiadas para negociar significados. De esta forma, el profesor necesitaba tener una adecuada formación para enfrentar cambios y, a su vez, ayudar a los alumnos en el aprendizaje (Frazer, 1979; Driver, 1988).

En este contexto, el aprendiz se sitúa en el centro del proceso y lo que él aprende va a depender de sus ideas previas, de las estrategias cognitivas de que dispone y, también, de 
sus propios propósitos e intereses (Driver, 1988). Frente a estas consideraciones, adoptar una concepción evolutiva, en cualquier planificación curricular, implicaba enfatizar las formas en las cuales se relacionan las ideas de los alumnos, lo que a su vez significaba respetar sus características evolutivas.

Se debe destacar, frente a ese contexto, que el constructivismo no es una teoría psicológica en un sentido estricto ni una teoría psicopedagógica que entregue una explicación completa, exacta y controlada empíricamente acerca de cómo aprenden los alumnos, y de la cual se pudiesen derivar prescripciones infalibles de cómo proceder mejor al abordar el proceso de enseñanza-aprendizaje. Lo que cabe enfatizar es que esta concepción rompía con la tradicional confrontación entre una metodología de enseñanza centrada en el alumno o en el profesor, proponiendo un adecuado ajuste entre los dos, con miras hacia el proceso de construcción del conocimiento. En este sentido, en lo que se refiere a la enseñanza de la química, eran importantes, en la sala de aula, las expresiones y discusiones de los alumnos sobre ideas químicas, dando así oportunidades para negociar los significados en busca de un consenso.

De esta manera, desde los años de 1980, se enfatiza la perspectiva constructivista del currículum, ya que el conocimiento científico es una construcción social, siendo producto colectivo de la humanidad. Para la concepción constructivista, la función de la educación debería ser la de promover el desarrollo y crecimiento personal de los alumnos. La realización de los aprendizajes por parte de ellos, sólo podía ser fuente creadora de desarrollo si les permitía un doble proceso, de socialización y de individualización, o sea, si permitía la construcción de una identidad personal en el marco de un contexto social y cultural determinado.

Al considerar, por ejemplo, esos aspectos en los temas propuestos por el movimiento CTS, se constata que corresponden a temas de relevancia social, con enfoque interdisciplinar que motive la interconexión entre los conceptos científicos y esos temas. Como ya fue mencionado, ese movimiento se caracteriza por la participación activa de los alumnos, incentivando el desarrollo de actitudes de toma de decisiones. Para esto, se proponen estrategias como: visitas a industrias y museos, estudios de caso sobre problemas reales de la sociedad, charlas, demostraciones, debates, realización de proyectos individuales y colectivos, investigaciones, experiencias de laboratorio, entre otros. Waddington (1980) señala que la participación directa de los alumnos en la experimentación ayudaría a desarrollar conceptos, lo que sería clave para promover un adecuado balance entre teoría y práctica.

\section{PROCEDIMIENTOS DE EVALUACIÓN}

El tema de la evaluación dentro del currículum es uno de los temas más complejos, ya que existen diferentes formas de abordarlo. Como ya ha sido mencionado al comienzo de este texto, tanto los objetivos como la evaluación constituyen dos aspectos que deben ser considerados en el mismo momento en que son establecidos los otros elementos del proceso de enseñanza-aprendizaje. O sea, cuando se determina lo que debe ser enseñado y lo que se espera que los alumnos aprendan, también se debe considerar cómo comprobar que los objetivos establecidos son cumplidos.

En la década de 1950, al relacionar los objetivos con la evaluación, se constata que uno de las funciones tradicionales del sistema educativo era la de clasificar y juzgar a las 
personas a través de pruebas que les permitirían, o no, el acceso a niveles superiores de educación o de trabajo. La evaluación se caracterizaba por medir los alumnos en relación a objetivos pre-definidos, utilizando un proceso de selección que los calificaba a través de pruebas (Ashman 1985). Quien evaluaba era el profesor, que consideraba productos finales, considerando la evaluación como un control.

Alrededor de los años de 1970, la evaluación pasa a ser vista como integrada al proceso educativo, o sea, ya no sólo como procedimiento de selección, pero sí de orientación, dirigido a mejorar a los alumnos en todos los ámbitos de su personalidad, considerando aspectos ambientales y personales en los cuales se desarrolla la acción educativa. Esta propuesta era considerada válida por los profesores, pero muchos de ellos no modifican sus hábitos o actitudes y continúan creyendo que el sistema funciona cuando la evaluación se limita a notas y exámenes.

Según Fernández (1988), la evaluación se presentaba, hasta ese entonces, como un triángulo de poder, donde tanto los conocimientos como los alumnos estaban al servicio del profesor, en lugar de que los profesores y los alumnos estuvieran al servicio de los conocimientos. Y dentro de esta concepción se mantuvo la evaluación por mucho tiempo: preocupados por la clasificación, selección y logro de los objetivos, centrándose la acción solamente en un cumplimiento burocrático.

Con la intensificación del enfoque constructivista, a partir de los años de 1980, fueron propuestos nuevos rumbos para la evaluación. Esta pasa a ser considerada como un elemento clave y fundamental que deja de ser exclusivamente del alumno y se refiere al propio proceso. Se caracterizaba, por un lado, por proponer un dialogo y reflexión sobre conflictos y dificultades de los alumnos, centrada en sus tareas, hábitos y actitudes. Corresponde principalmente a una evaluación procesual y no a aquella que clasifica o selecciona. O sea, orienta el proceso de aprendizaje de los alumnos y el auto-aprendizaje de los profesores de manera continua.

Esto implicaba que se debe comenzar por el conocimiento inicial de la situación de los alumnos, determinando los progresos y dificultades del proceso de aprendizaje y, a partir de esto, establecer la ayuda pedagógica necesaria. Correspondía a una evaluación formativa que valorizaba lo que el alumno asimilaba durante el proceso de enseñanzaaprendizaje. Contrario a la evaluación sumativa, centrada en la verificación de productos finales, la evaluación formativa buscaba detectar si los objetivos se concretizaban en forma de capacidades que fueran alcanzadas progresivamente (evaluación de proceso).

Finalmente, resultaba prioritario destacar una evaluación que fuera formativa, integral y continua, dando prioridad a los procesos. Estos son, por lo tanto, los atributos que debían caracterizar la evaluación en propuestas curriculares de la época.

Frente a estas consideraciones expuestas, se puede constatar que la educación científica, y en particular la educación química, presentaron un significativo desarrollo en ese periodo, básicamente dirigido a la necesidad de pasar de la instrucción como capacitación intelectual, a una educación como formación integral. Esa tendencia, incorporaba los siguientes aspectos:

- difusión de diferentes corrientes relacionadas con el desarrollo de la química y la instauración de nuevos contenidos;

- preocupación por aspectos relativos a cómo es elaborado el conocimiento (tradicionalmente llamado procesos científicos); 
- interés por lo cognitivo y lo social, incorporando aspectos constructivistas de manera socialmente contextualizada.

Estos aspectos demuestran un proceso de evolución vivido por la educación científica, al considerar un cambio en la concepción curricular, enfatizando la construcción social del conocimiento científico.

A partir de las consideraciones expuestas en este trabajo, podemos analizar de qué manera se realiza la práctica de los profesores de química actualmente. Queda, ahora, pendiente la tarea de estudiar de qué manera ha evolucionado la educación química en las dos últimas décadas, hasta los tiempos actuales.

\section{AGRADECIMIENTOS}

Mis agradecimientos a la Profa. Dra.Roseli Pacheco Schnetzler por sus orientaciones y enseñanzas durante el proceso de esta investigación.

\section{REFERENCIAS BIBLIOGRÁFICAS}

Ashman, A. (1985). Chemistry in School - past, present and future: Part I. School Science Review 66 (237), p. 696-703.

Blizzard, A.C., Humphreys, D.A., Hunter, B. y Martin, R. (1975). Chemistry Core Topics. Chem 13 News (66), p. 2-5.

Buchweitz, B. (1985). O uso de mapas conceituais na análise do currículo. Ensino na Universidade. EDUNISUL: Porto Alegre. p. 20-32.

Caamaño, A. (1988). Tendencias actuales en el currículum de ciencias. Enseñanza de las Ciencias 6 (3), p. 265-277.

Driver, R. (1988). Un enfoque constructivista para el desarrollo del currículum de ciencias. Enseñanza de las Ciencias 6 (2), p. 108-120.

Faúndez-Vallejos, N. (1993). Concepções de professores sobre a implementação do programa oficial de Química na escola secundária chilena. Dissertação de Mestrado. Campinas: Faculdade de Educação, Universidade Estadual de Campinas.

Fernández, M. (1988). La profesionalización del Docente. Madrid: Editorial Escuela Española S.A.

Frazer, M. (1984). The future of science education. British Association for the Advancement of Science, p. 1-14.

. (1979).Trends in Chemical Education. Kemia-Kemi 6 (4), p. 147-150.

Gil, D., Carrascosa, J., Furio, C. y Martinez Torregrosa, J. (1991). La enseñanza de las ciencias en la Educación Secundaria. Barcelona: Editorial HORSORI.

Gillespie, R. y Humphreys, D. (1972). Chemistry Core Curriculum. Chem 13 News (43), p. 9-12.

Hernández, F. (1990). El currículo. Cuadernos de Pedagogía (185), p. 12-14.

Hodson, D. y Reid, D. (1988). Science for all: A curriculum developer's checklist. School Science Review 69 (249), p. 821-826.

Hostettler, J.D. (1985). Science for citizens: a plan with a purpose. Journal of Chemical Education 162 (9), p. 764-765.

Kelly, A. (1981). O Currículo: Teoria e Prática. São Paulo: Harper \& Row do Brasil.

Kempa, R. (1986). Assessment in Science. London: Cambridge University Press.

Klopfer, L. (1971). Evaluation of Learning in Science. En Bloom, B., Hastings, T. y Madaus, G. 
Handbook on Formative and Summative Evaluation of Student Learning. New York: Mc GrawHill Book Company. Cap. 18, p. 561-641.

Kornhauser, A. (1980). Chemical Education - Today's challenges for tomorrow's development. En Kornhauser, A., Rao, C.N.R., Waddington, D. (org.). Chemical Education for the Seventies, IUPAC. New York: Pergamon Press, p. 308-327.

Matthews, M. (1990). History, Philosophy and Science teaching: a rapprochement. Studies in Science Education 18, p. 25-51.

Moreira, M. y Axt, R. (1987). Referenciais para a análise e planejamento de currículo em ensino de ciências. Ciência e Cultura 39 (3), p. 250-258.

Pode, J. (1967). Los cursos CBA y CHEMS: una crítica apreciativa. Revista Iberoamericana de Educación Química (2), p. 58-64.

Santos, M. (1991). Elementos contextuais na emergência de um movimento pedagógico. En Mudança Conceitual na Sala de Aula - Um desafio pedagógico. Lisboa: Livro Horizonte. p. 26-47.

Waddington, D. (1980). Chemical Education: Changes Worldwide. En Kornhauser, A.; Rao, C.N.R. y Waddington, D. (org.). Chemical Education in the Seventies, IUPAC. New York: Pergamon Press, p. 1-29.

Ware, S., Heikkinen, H. y Lippinott, W. (1986). The CHEMCOM. Philosophy and approach. International Newsletter on Chemical Education (26), p. 10-12. 
